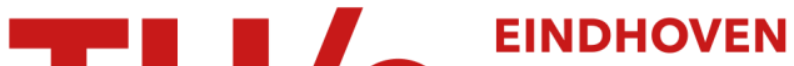 \\ UNIVERSITY OF \\ TECHNOLOGY
}

\section{On the numerical integration of interface elements}

\section{Citation for published version (APA):}

Schellekens, J. C. J., \& Borst, de, R. (1993). On the numerical integration of interface elements. International Journal for Numerical Methods in Engineering, 36(1), 43-66. https://doi.org/10.1002/nme.1620360104

DOI:

10.1002/nme.1620360104

Document status and date:

Published: 01/01/1993

\section{Document Version:}

Publisher's PDF, also known as Version of Record (includes final page, issue and volume numbers)

\section{Please check the document version of this publication:}

- A submitted manuscript is the version of the article upon submission and before peer-review. There can be important differences between the submitted version and the official published version of record. People interested in the research are advised to contact the author for the final version of the publication, or visit the $\mathrm{DOI}$ to the publisher's website.

- The final author version and the galley proof are versions of the publication after peer review.

- The final published version features the final layout of the paper including the volume, issue and page numbers.

Link to publication

\section{General rights}

Copyright and moral rights for the publications made accessible in the public portal are retained by the authors and/or other copyright owners and it is a condition of accessing publications that users recognise and abide by the legal requirements associated with these rights.

- Users may download and print one copy of any publication from the public portal for the purpose of private study or research.

- You may not further distribute the material or use it for any profit-making activity or commercial gain

- You may freely distribute the URL identifying the publication in the public portal.

If the publication is distributed under the terms of Article $25 \mathrm{fa}$ of the Dutch Copyright Act, indicated by the "Taverne" license above, please follow below link for the End User Agreement:

www.tue.nl/taverne

Take down policy

If you believe that this document breaches copyright please contact us at:

openaccess@tue.nl

providing details and we will investigate your claim. 


\title{
ON THE NUMERICAL INTEGRATION OF INTERFACE ELEMENTS
}

\author{
J. C. J. SCHELLEKENS AND R. DE BORST \\ Delft University of Technology, Department of Civil Engineering, TNO Building and Construction Research, \\ P.O. Box. 5048, 2600 GA Delft, The Netherlands
}

\begin{abstract}
SUMMARY
Eigenmode analyses of the element stiffness matrices have been used to assess the impact of the applied integration scheme on the stress predictions of two- and three-dimensional plane interface elements. It is demonstrated that large stress gradients over the element and coupling of the individual node-sets of the interface element may result in an oscillatory type of response. For line elements and linear plane interface elements the performance can be improved by using either a nodal lumping scheme or Newton-Cotes or Lobatto integration schemes instead of the more traditional Gauss scheme. For quadratic interface elements the same holds true except for a nodal lumping scheme.
\end{abstract}

\section{INTRODUCTION}

Interface elements are a powerful tool in the modelling of geometrical discontinuities in different kinds of structures. In finite element analysis of civil engineering structures a large variety of applications for interface elements is present. Interface elements can be used to model soil reinforcement interaction, ${ }^{1}$ to model the intermediate layer between rock and concrete, e.g. in arch dam analysis ${ }^{2-4}$, or in the analysis of rock joints. ${ }^{5,6}$ Applications in concrete mechanics cover the modelling of discrete cracking, ${ }^{7,8}$ aggregate interlock ${ }^{9}$ and bond between concrete and reinforcement. ${ }^{10-14}$ In rubber parts, interface elements can be of importance when disintegration of rubber and texture is concerned, e.g. in conveyor belts. Furthermore, interface elements are suited to model delamination in layered composite structures ${ }^{15,16}$ or frictional contact in forming processes. $^{17}$

Interface elements can be divided into two elementary classes. The first class contains the continuous interface elements (line, plane and shell interfaces), ${ }^{1-9,11-18,20-23}$ whereas the second class of elements contains the nodal or point interface elements, ${ }^{7,10,21}$ which, to a certain extent, are identical to spring elements. In this contribution we shall only consider the numerically and lumped integrated continuous interface elements, since nodal interfaces are integrated explicitly.

A basic requirement of interface elements is that during the elastic stage of the loading process no significant additional deformations occur due to the presence of these elements in the finite element model. Therefore, a sufficiently high initial dummy stiffness has to be supplied for the interface elements. Depending upon the applied numerical integration scheme, this high dummy stiffness may result in undesired spurious oscillations of the stress field. In this paper the impact of Gauss, Newton-Cotes, Lobatto and lumped integration schemes on the stress prediction in interfaces is investigated for three-dimensional linear and non-linear analyses. Eigenvalue analyses of the linear elastic and non-linear element stiffness matrices have been carried out to explain the observed oscillatory performance of interface elements. Since we shall focus on

0029-5981/93/010043-24\$17.00

(C) 1993 by John Wiley \& Sons, Ltd.
Received 6 September 1991 Revised 1 April 1992 
threc-dimensional applications, the results from the two-dimensional analyses are discussed only briefly.

\section{FINITE ELEMENT FORMULATION}

As mentioned in the introduction, the element stiffness matrix of line and plane interface elements can either be assembled by numerical or by lumped integration. The difference stems from the fact that in numerically integrated interfaces the traction vs. relative displacement relations are evaluated along an interpolated displacement field in the integration points, whereas the lumped interfaces evaluate the relation at the individual node sets.

\subsection{Numerically integrated interface elements}

Consider an $m$-noded plane interface as in Figure 1. Each node has three translational degrees of freedom, which leads to an element nodal displacement vector $v$

$$
\boldsymbol{v}=\left(v_{n}^{1}, v_{n}^{2}, \ldots, v_{n}^{m}, v_{s}^{1}, v_{s}^{2}, \ldots, v_{s}^{m}, v_{t}^{1}, v_{t}^{2}, \ldots, v_{t}^{m}\right)^{\mathbf{T}}
$$

where $n$ denotes the direction normal to the interface surface and $s$ and $t$ denote the directions tangential to the interface surface. The continuous displacement field is denoted as

$$
\mathbf{u}=\left(u_{n}^{u}, u_{n}^{l}, u_{s}^{u}, u_{s}^{l}, u_{t}^{u}, u_{t}^{l}\right)^{\mathrm{T}}
$$

where the superscripts $u$ and $l$ indicate the upper and lower side of the interface, respectively. With the aid of the interpolation polynomials $\mathbf{n}=\left(N_{1}, N_{2}, \ldots, N_{m / 2}\right)$, the relation between the continuous displacement field and the nodal displacement vector is derived as

$$
\mathbf{u}=\mathbf{H v}
$$

in which $\mathbf{H}$ contains the interpolation polynomials according to

$$
\mathbf{H}=\left[\begin{array}{llllll}
\mathbf{n} & 0 & 0 & 0 & 0 & 0 \\
0 & \mathbf{n} & 0 & 0 & 0 & 0 \\
0 & 0 & \mathbf{n} & 0 & 0 & 0 \\
0 & 0 & 0 & \mathbf{n} & 0 & 0 \\
0 & 0 & 0 & 0 & \mathbf{n} & 0 \\
0 & 0 & 0 & 0 & 0 & \mathbf{n}
\end{array}\right]
$$

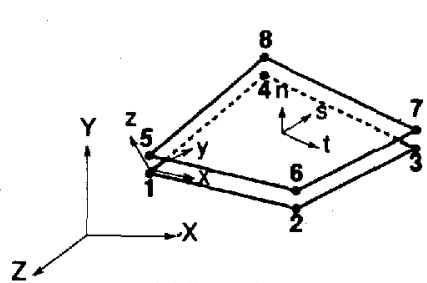

(a) linear plane interface

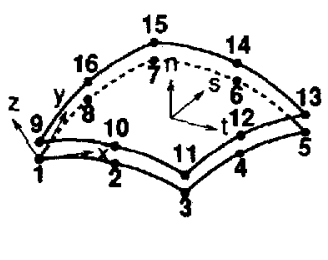

(b) quadratic plane interface

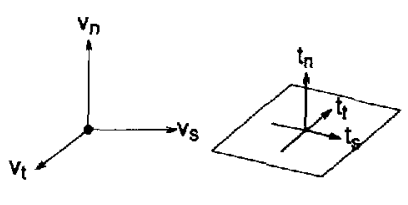

(c) nodal displacements

(d) tractions

Figure 1. Linear and quadratic plane interface elements 
To relate the continuous displacement field to the relative displacements, an operator matrix $\mathbf{L}$ is introduced

$$
\mathbf{L}=\left[\begin{array}{rrrrrr}
-1 & +1 & 0 & 0 & 0 & 0 \\
0 & 0 & -1 & +1 & 0 & 0 \\
0 & 0 & 0 & 0 & -1 & +1
\end{array}\right]
$$

When the relative displacement vector $\Delta \mathbf{u}$ is defined as $\Delta \mathbf{u}=\left(\Delta u_{n}, \Delta u_{s}, \Delta u_{t}\right)^{\mathrm{T}}$, we obtain

$$
\Delta \mathbf{u}=\mathbf{L u}
$$

The relation between nodal displacements and relative displacements for continuous elements is now derived from equations (3) and (6) as

$$
\Delta \mathbf{u}=\mathbf{L H} \mathbf{v} \rightarrow \Delta \mathbf{u}=\mathbf{B v}
$$

where relative displacement vs. nodal displacement matrix $\mathbf{B}$ reads

$$
\mathbf{B}=\left[\begin{array}{rrrrrr}
-\mathbf{n} & \mathbf{n} & 0 & 0 & 0 & 0 \\
0 & 0 & -\mathbf{n} & \mathbf{n} & 0 & 0 \\
0 & 0 & 0 & 0 & -\mathbf{n} & \mathbf{n}
\end{array}\right]
$$

Since we consider an element in which the local co-ordinate systems in the integration points coincide with the global co-ordinate system, no transformations are necessary. For an arbitrary oriented interface element, the matrix $\mathbf{B}$ has to be transformed to the local tangential co-ordinate system of the integration point or node set.

When the matrix $\mathbf{D}$ is used to denote the relation that describes the constitutive behaviour of the interface element

$$
\mathbf{D}=\left[\begin{array}{ccc}
d_{n} & 0 & 0 \\
0 & d_{s} & 0 \\
0 & 0 & d_{t}
\end{array}\right]
$$

the traction vs. relative displacement relation becomes

$$
\mathbf{t}=\mathbf{D} \Delta \mathbf{u}
$$

in which $\mathrm{t}=\left(t_{n}, t_{s}, t_{t}\right)^{\mathrm{T}}$ represents the traction vector.

The linear element stiffness matrix $\mathbf{K}$ can now be obtained using the standard procedure of minimizing the total amount of potential energy. ${ }^{19}$ The amount of internal work done in the interface element equals

$$
U=\frac{1}{2} \int_{A} \Delta \mathbf{u}^{\mathrm{T}} \mathrm{t} \mathrm{d} A
$$

which, after invoking equations (7) and (10), results in

$$
U=\frac{1}{2} \mathbf{v}^{\mathrm{T}} \int_{A} \mathbf{B}^{\mathrm{T}} \mathbf{D B} \mathrm{d} A \boldsymbol{v}
$$

The amount of external work is given by

$$
W=-\mathbf{v}^{\mathrm{T}} \mathbf{f}
$$


with $\mathbf{f}$ the external force vector. After variation of the total potential energy $(U+W)$ with respect to the nodal displacement vector we obtain

$$
\mathbf{K} \mathbf{v}=\mathbf{f}
$$

where the stiffness matrix $\mathbf{K}$ equals

$$
\mathbf{K}=\int_{A} \mathbf{B}^{\mathrm{T}} \mathbf{D B} \mathrm{d} A
$$

For numerically integrated interface elements, the integral in equation (15) is replaced by an integration over the iso-parametric co-ordinates $\xi$ and $\eta$ :

$$
\mathbf{K}=\int_{\xi=-1}^{\xi=+1} \int_{\eta=-1}^{\eta=+1} \mathbf{B}^{\mathbf{T}} \mathbf{D B} \operatorname{det} \mathbf{J} \mathrm{d} \xi \mathrm{d} \eta
$$

with det $\mathbf{J}$ the determinant of the Jacobian matrix. For line interfaces the interpolation functions $N_{i}$ are independent of $\eta$ and equation (16) reduces to

$$
\mathbf{K}=b \int_{\xi=-1}^{\xi=+1} \mathbf{B}^{\mathrm{T}} \mathbf{D B}\left[\left(\frac{\partial x}{\partial \xi}\right)^{2}+\left(\frac{\partial y}{\partial \xi}\right)^{2}\right]^{1 / 2} \mathrm{~d} \xi
$$

where $b$ is the width of the interface. If, for example, we use a $2 \times 2$ Gauss integration scheme for the assembly of the element stiffness matrix of a linear eight-noded plane interface with surface $A$, the result is

$$
\mathbf{K}=\frac{1}{36} A\left[\begin{array}{ccc}
\mathbf{K}_{n} & \mathbf{0} & \mathbf{0} \\
\mathbf{0} & \mathbf{K}_{s} & \mathbf{0} \\
\mathbf{0} & \mathbf{0} & \mathbf{K}_{t}
\end{array}\right]
$$

where each $8 \times 8$ submatrix has the form

$$
\mathbf{K}_{i}=\left[\begin{array}{rrrrrrrr}
4 d_{i} & 2 d_{i} & d_{i} & 2 d_{i} & -4 d_{i} & -2 d_{i} & -d_{i} & -2 d_{i} \\
2 d_{i} & 4 d_{i} & 2 d_{i} & d_{i} & -2 d_{i} & -4 d_{i} & -2 d_{i} & -d_{i} \\
d_{i} & 2 d_{i} & 4 d_{i} & 2 d_{i} & -d_{i} & -2 d_{i} & -4 d_{i} & -2 d_{i} \\
2 d_{i} & d_{i} & 2 d_{i} & 4 d_{i} & -2 d_{i} & -d_{i} & -2 d_{i} & -4 d_{i} \\
-4 d_{i} & -2 d_{i} & -d_{i} & -2 d_{i} & 4 d_{i} & 2 d_{i} & d_{i} & 2 d_{i} \\
-2 d_{i} & -4 d_{i} & -2 d_{i} & -d_{i} & 2 d_{i} & 4 d_{i} & 2 d_{i} & d_{i} \\
-d_{i} & -2 d_{i} & -4 d_{i} & -2 d_{i} & d_{i} & 2 d_{i} & 4 d_{i} & 2 d_{i} \\
-2 d_{i} & -d_{i} & -2 d_{i} & -4 d_{i} & 2 d_{i} & d_{i} & 2 d_{i} & 4 d_{i}
\end{array}\right]
$$

for $i=n, s$ and $t$.

\subsection{Lumped integrated interface elements}

The major difference between lumped and numerically integrated interface elements is the use of relative displacements at the isolated node sets instead of an interpolated relative displacement 
field in integration points. Again we introduce the element vector of equation (1)

$$
\mathbf{v}=\left(v_{n}^{1}, v_{n}^{2}, \ldots, v_{n}^{m}, v_{s}^{1}, v_{s}^{2}, \ldots, v_{s}^{m}, v_{t}^{1}, v_{t}^{2}, \ldots, v_{t}^{m}\right)^{\mathrm{T}}
$$

and the relative displacements vs. nodal displacement relation

$$
\Delta \mathbf{u}=\mathbf{B}_{i s} \boldsymbol{v}
$$

with $\mathbf{B}_{i s}$ the relative displacement-nodal displacement matrix for a node set is. For a lumped interface element, elaborating the integral in equation (15) results in a summation over the element node sets. Hence,

$$
\mathbf{K}=\sum_{i s=1}^{n s} \mathbf{B}_{i s}^{\mathrm{T}} \mathbf{D}_{i s} \mathbf{B}_{i s} A_{i s}
$$

where $n s$ denotes the number of node sets and $A_{i s}$ is the surface contribution of node set is. Since the traction vs. relative displacement relation is evaluated in the individual node sets instead of in the integration points, the matrix $\mathbf{B}_{i s}$ is obtained as

$$
\mathbf{B}_{i s}=\left[\begin{array}{rrrrrr}
-1 & +1 & 0 & 0 & 0 & 0 \\
0 & 0 & -1 & +1 & 0 & 0 \\
0 & 0 & 0 & 0 & -1 & +1
\end{array}\right]
$$

With the sequence of element degrees of freedom as in equation (1) this results in the following nodal displacement vs. relative displacement matrix $\mathbf{B}_{1}$ for the first node set of a linear plane interface element

$$
\mathbf{B}_{1}=\left[\begin{array}{rrrrrrrrrrrrrrrrrrrrrrrr}
-1 & 0 & 0 & 0 & 1 & 0 & 0 & 0 & 0 & 0 & 0 & 0 & 0 & 0 & 0 & 0 & 0 & 0 & 0 & 0 & 0 & 0 & 0 & 0 \\
0 & 0 & 0 & 0 & 0 & 0 & 0 & 0 & -1 & 0 & 0 & 0 & 1 & 0 & 0 & 0 & 0 & 0 & 0 & 0 & 0 & 0 & 0 & 0 \\
0 & 0 & 0 & 0 & 0 & 0 & 0 & 0 & 0 & 0 & 0 & 0 & 0 & 0 & 0 & 0 & -1 & 0 & 0 & 0 & 1 & 0 & 0 & 0
\end{array}\right]
$$

The surface contributions $A_{i s}$ of the node sets are determined from:

$$
\mathrm{d} A_{i p}=\operatorname{det} \mathbf{J} \mathrm{d} \xi \mathrm{d} \eta
$$

and

$$
A_{i s}=\sum_{i p=1}^{n i p} N_{i s, i p} \mathrm{~d} A_{i p}
$$

where $N_{i s, i p}$ is the value of the interpolation polynomial of node-set is at integration point ip. As an example, the stiffness matrix is given for an eight-noded linear plane interface with surface $A$ :

$$
\mathbf{K}=\frac{1}{4} A\left[\begin{array}{ccc}
\mathbf{K}_{n} & \mathbf{0} & \mathbf{0} \\
\mathbf{0} & \mathbf{K}_{s} & \mathbf{0} \\
\mathbf{0} & \mathbf{0} & \mathbf{K}_{t}
\end{array}\right]
$$


in which the $8 \times 8$ submatrix $\mathbf{K}_{i}$ has the form

$$
\mathbf{K}_{i}=\left[\begin{array}{rrrrrrrr}
d_{i} & 0 & 0 & 0 & -d_{i} & 0 & 0 & 0 \\
0 & d_{i} & 0 & 0 & 0 & -d_{i} & 0 & 0 \\
0 & 0 & d_{i} & 0 & 0 & 0 & -d_{i} & 0 \\
0 & 0 & 0 & d_{i} & 0 & 0 & 0 & -d_{i} \\
-d_{i} & 0 & 0 & 0 & d_{i} & 0 & 0 & 0 \\
0 & -d_{i} & 0 & 0 & 0 & d_{i} & 0 & 0 \\
0 & 0 & -d_{i} & 0 & 0 & 0 & d_{i} & 0 \\
0 & 0 & 0 & -d_{i} & 0 & 0 & 0 & d_{i}
\end{array}\right]
$$

where $i$ can be $n, s$, and $t$. It is noted that no coupling of degrees of freedom exists between the individual node sets.

\section{INTEGRATION SCHEMES AND ELEMENT PERFORMANCE}

\subsection{Introduction}

In finite element analysis the surface or line integral to determine the element stiffness matrix is replaced by a weighted sum as

$$
\mathbf{K}=\sum_{i p=1}^{n i p} \alpha_{i p} A \mathbf{B}_{i p}^{\mathrm{T}} \mathbf{D}_{i p} \mathbf{B}_{i p}
$$

where the values of $\mathbf{B}_{i p}$ and the weight factor $\alpha_{i p}$ are dependent on the applied integration scheme and $A$ is the surface of the element. For numerical integration of continuum elements the accurate Gauss scheme is commonly used, whereas thickness integration of shells is usually performed using a Simpson integration rule. However, in recent publications $1,2,7,20-22$ it was found that under certain conditions, the application of Gaussian integration to interface elements leads to oscillatory traction profiles owing to spurious kinematic element performance. In this section we shall examine the effects of Gauss, Newton-Cotes/Lobatto and nodal lumping schemes on the behaviour of plane interface elements in $3 \mathrm{D}$-analyses of a notched beam and a soil reinforcement system. For the different integration schemes the locations of the integration points and the corresponding weight factors are collected in Figure 2. It is noted that Newton-Cotes and Lobatto schemes are identical for the cases of two and three sampling points, respectively. This is not so for higher-order quadrature, but since, in this paper, we shall restrict the discussion to linear and quadratic interface elements we shall henceforth refer to this integration scheme as Newton-Cotes/Lobatto.

\subsection{Three-dimensional linear elastic analysis of a notched beam}

We shall start with a discussion on the impact of different integration schemes on the results of an analysis of a three-dimensional notched beam. ${ }^{20.21}$ When interface elements are included in a finite element model the major prerequisite is that no additional deformations are introduced in the elastic stage. For this reason sufficiently high stiffness values for the interfaces have to be inserted. In the notched beam of Figure 3 interface elements are included in front of the notch to 
model the possible development of discrete crack. The question is how large the initial stiffness of the interface should be taken such that on the one hand additional deformations are negligible and on the other hand a realistic traction profile is obtained. Different analyses have been carried out using linear and quadratic elements. The stiffness values $d_{n}$ for the interfaces ranged from $10^{+3} \mathrm{~N} / \mathrm{mm}^{3}$ to $10^{+5} \mathrm{~N} / \mathrm{mm}^{3}$. A Young's modulus $E=20000 \mathrm{~N} / \mathrm{mm}^{2}$ and a Poisson's ratio

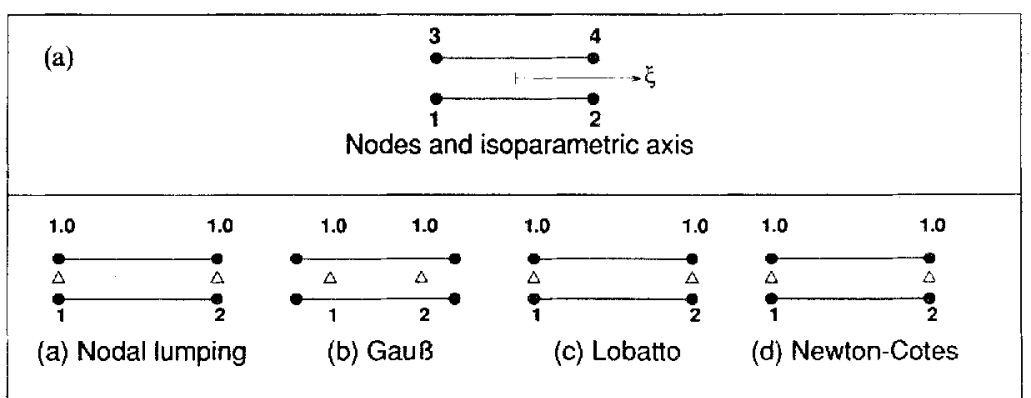
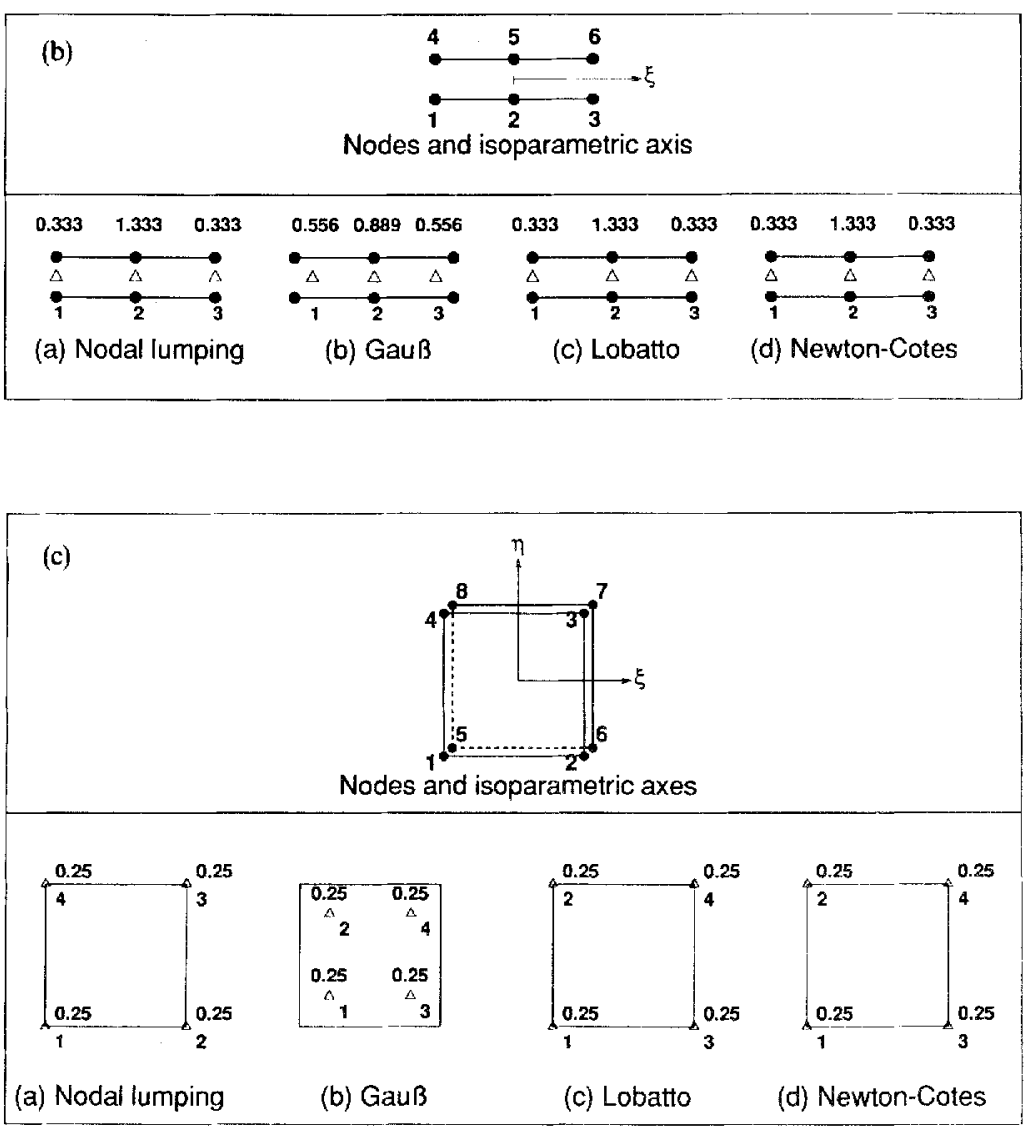

Figure 2. $(\mathrm{a}-\mathrm{c})$ (continued) 


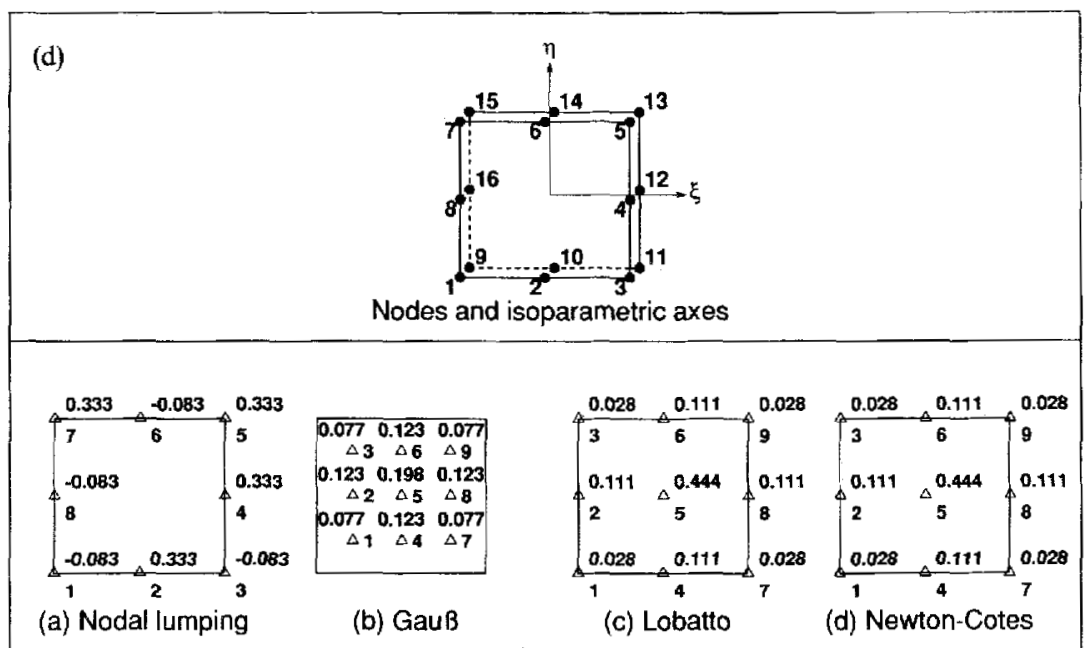

Figure 2. Locations and weight factors for integration points. (a) Linear line interface elements; (b) Quadratic line interface elements; (c) Lincar plane interface elements; (d) Quadratic plane interface elements

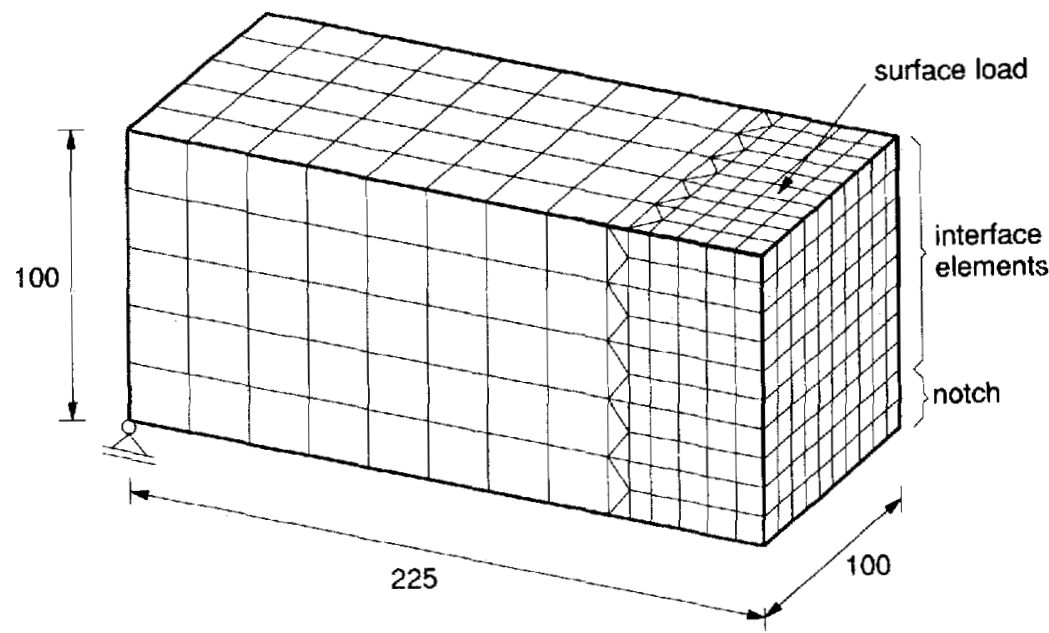

Figure 3. Finite element model of a three-dimensional concrete beam

$v=0.2$ were used for the continuum elements. Since the fracture problem is of pure mode-I type the values of the shear stiffnesses $d_{s}$ and $d_{t}$ are irrelevant.

The beam is loaded in the vertical direction by a surface load on the second row of elements from the symmetry plane as indicated in Figure $3\left(F_{\text {tot }}=1 \mathrm{kN}\right)$. The results for the linear eight-noded plane interface element fully agree with the results from a two-dimensional analysis ${ }^{21}$ in the sense that for $2 \times 2$ Gauss integration oscillations occur in the vertical direction for stiffness values higher than $10^{+3} \mathrm{~N} / \mathrm{mm}^{3}$, while $2 \times 2$ Newton-Cotes/Lobatto integration and the nodal lumping scheme produce satisfactory results for all applied stiffness values (Figures 4 and 5). In the horizontal direction the traction profiles are free from oscillations, since there is no influence 

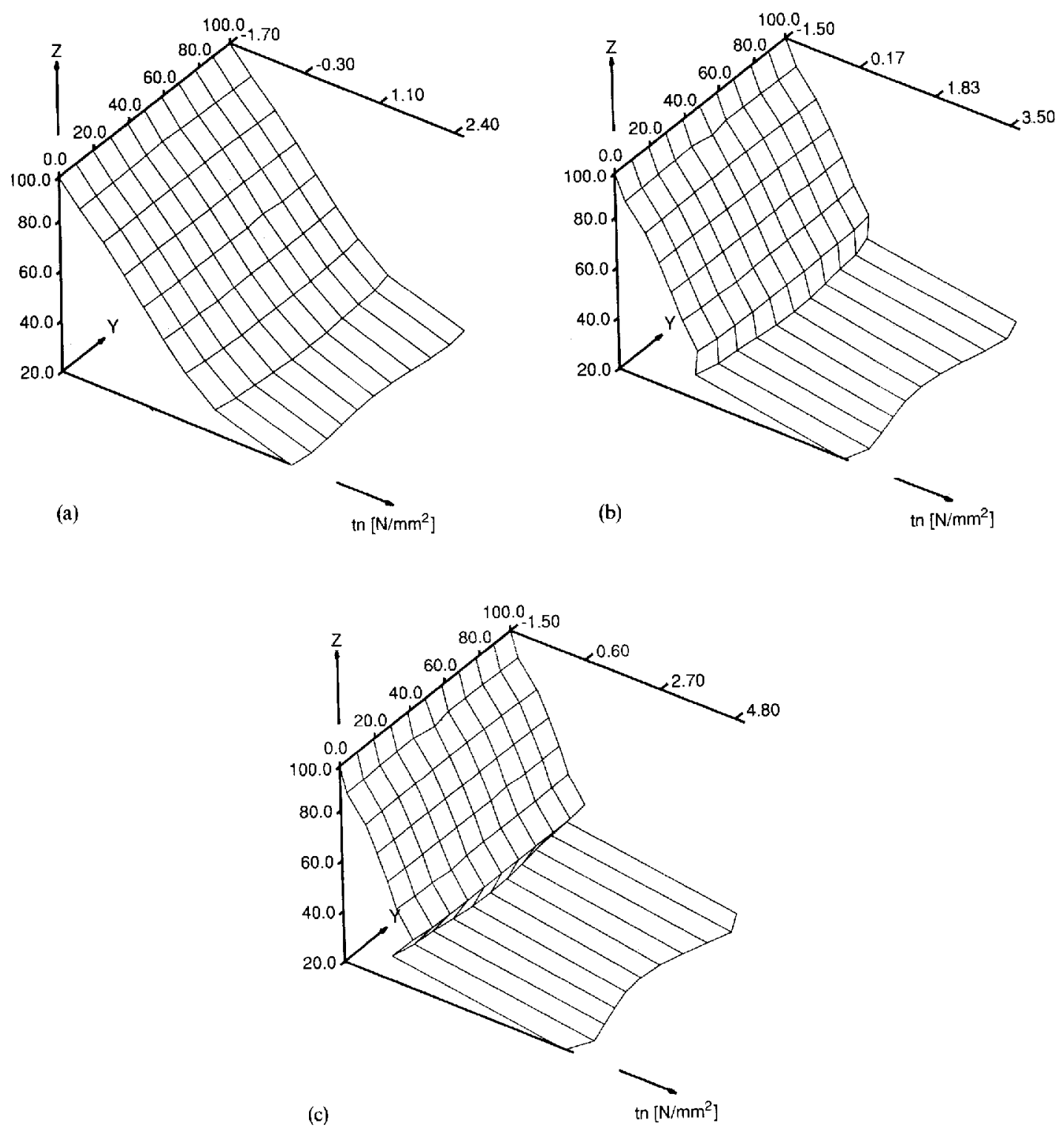

Figure 4. Traction profiles for linear plane interface elements with $2 \times 2$ Gauss integration: (a) $d_{n}=10^{+3} ;\left(\right.$ b) $d_{n}=10^{+4}$; (c) $d_{n}=10^{+5}$

of the notch singularity and no traction gradients exist. The traction profiles for the mesh with quadratic elements are presented in Figures 6 8. From Figures 4-8 the following observations are made:

- Satisfactory results are obtained for all integration schemes for $d_{n}=10^{+3} \mathrm{~N} / \mathrm{mm}^{3}$.

- Beyond stiffness values of $10^{+3} \mathrm{~N} / \mathrm{mm}^{3}$ application of the Gauss integration scheme leads to oscillatory results. 

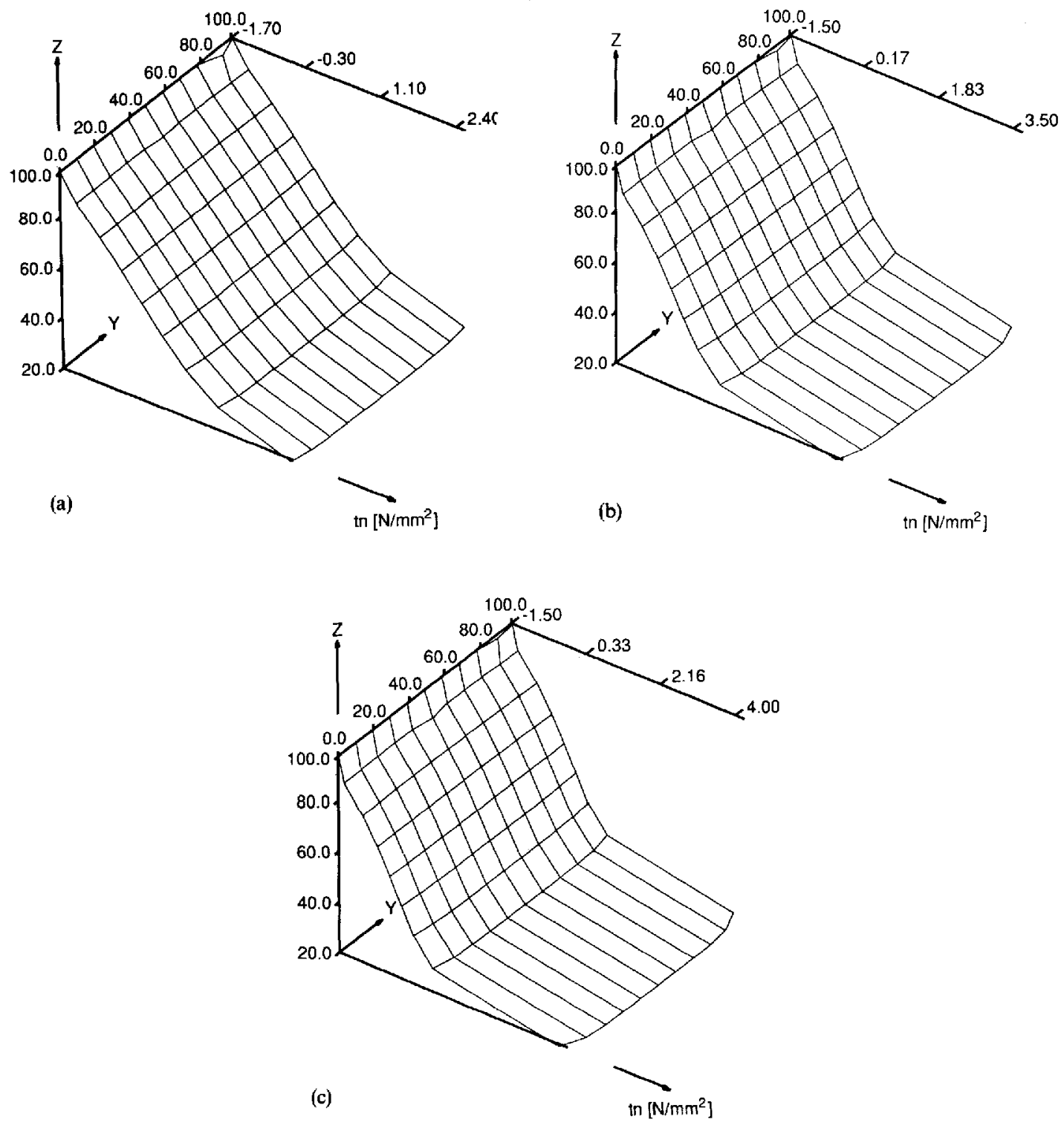

Figure 5. Traction profiles for linear plane interface elements with $2 \times 2$ Newton-Cotes/Lobatto integration: (a) $d_{n}=10^{+3} ;$ (b) $d_{n}=10^{+4} ;$ (c) $d_{n}=10^{+5}$

- Application of exact Newton-Cotes/Lobatto integration leads to correct results for the whole range of stiffness values.

- The lumped integration scheme yields proper results for linear plane interface elements, but not for quadratic plane interface elements. This is because the negative surface contributions of the corner nodes of quadratic elements then introduce negative diagonal terms in the element stiffness matrix. This leads to an ill-conditioned system of equations which is solved inaccurately with an LDU-decomposition without pivoting as has been done here. 

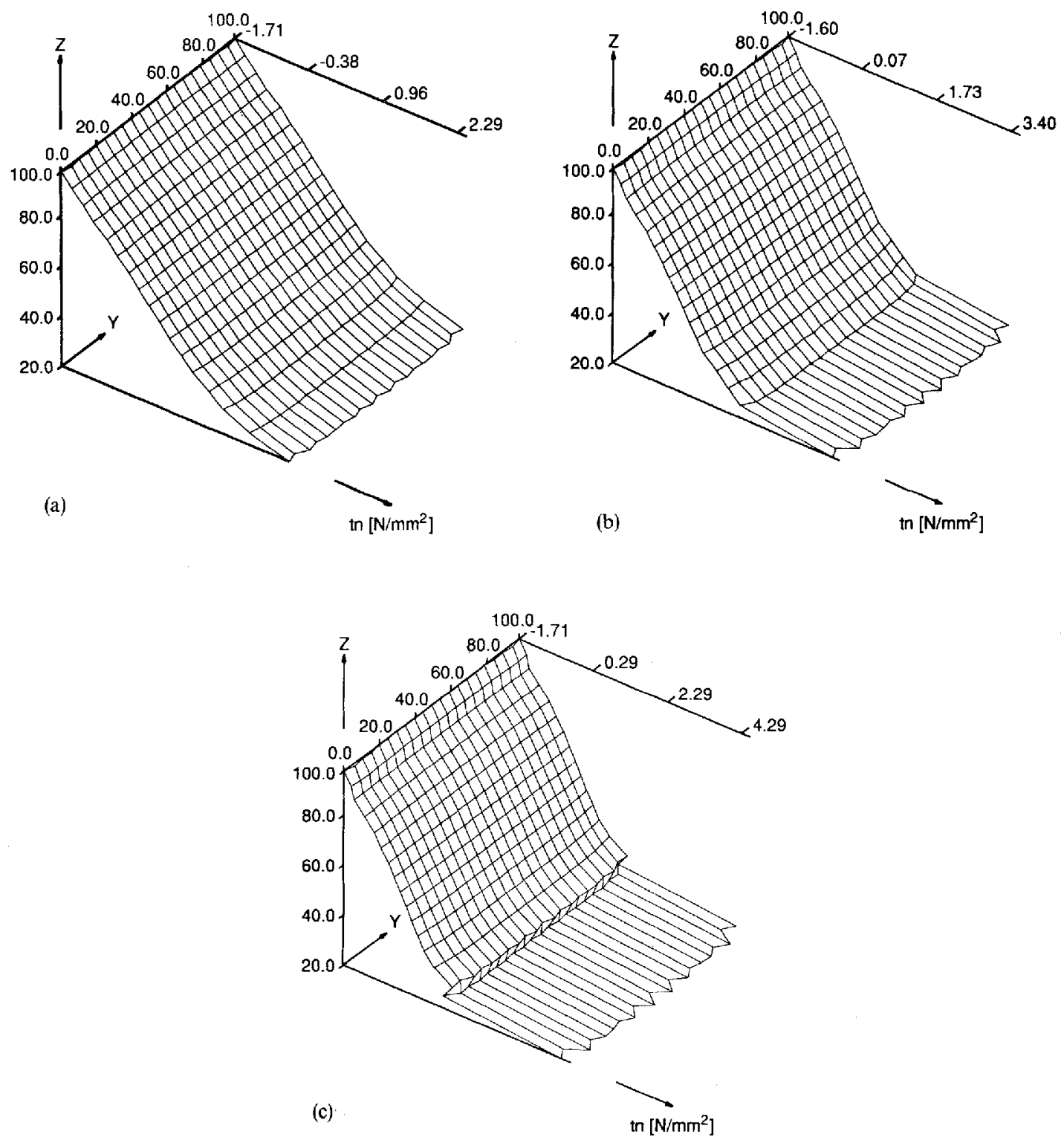

Figure 6. Traction profiles for quadratic plane interface elements with $3 \times 3$ Gauss integration: (a) $d_{n}=10^{+3}$; (b) $d_{n}=10^{+4}$; (c) $d_{n}=10^{+5}$

When, in case of Newton-Cotes/Lobatto integration, a scheme is applied with more sampling points than necessary (over-integration) oscillating traction distributions are again obtained. This is because four-point and higher-order Lobatto or Newton-Cotes schemes result in a stiffness matrix for the interface element that is identical to that which results from a three-point Gauss scheme, and therefore, yields no improvement with respect to the element performance. Also over-integration and under-integration using a Gauss scheme do not result in improvements with 

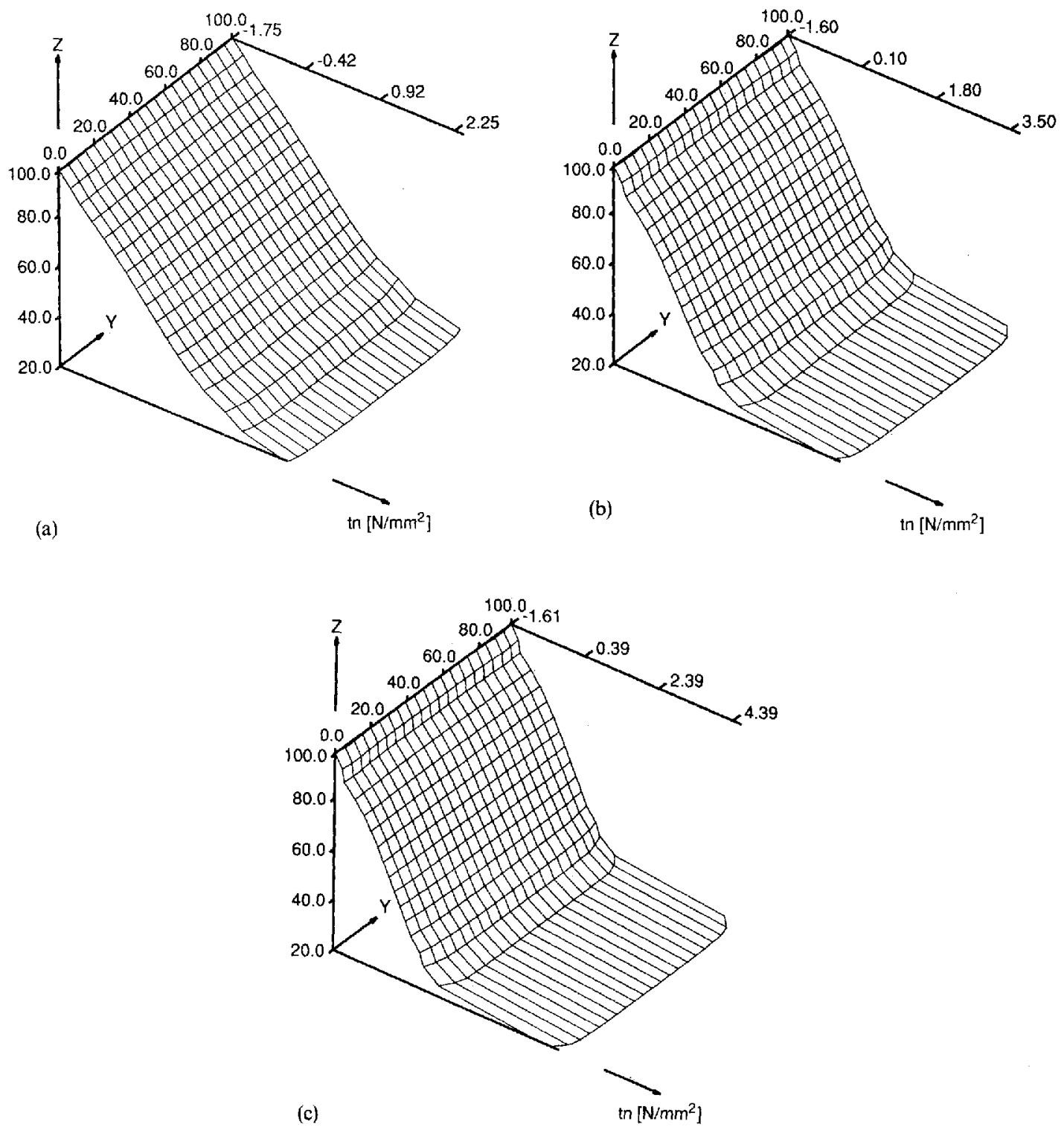

Figure 7. Traction profiles for quadratic plane interface elements with $3 \times 3$ Newton-Cotes/Lobatto integration: (a) $d_{n}=10^{+3}$; (b) $d_{n}=10^{+4}$; (c) $d_{n}=10^{+5}$

respect to the oscillations in the traction profiles that are observed for the standard Gauss integration.

\subsection{Non-linear analysis of soil-reinforcement interaction}

In addition to the mode- $I$ analyses of the concrete beam the performance of the interface elements has also been examined for pure mode-II behaviour. ${ }^{1,21}$ The problem that is considered 

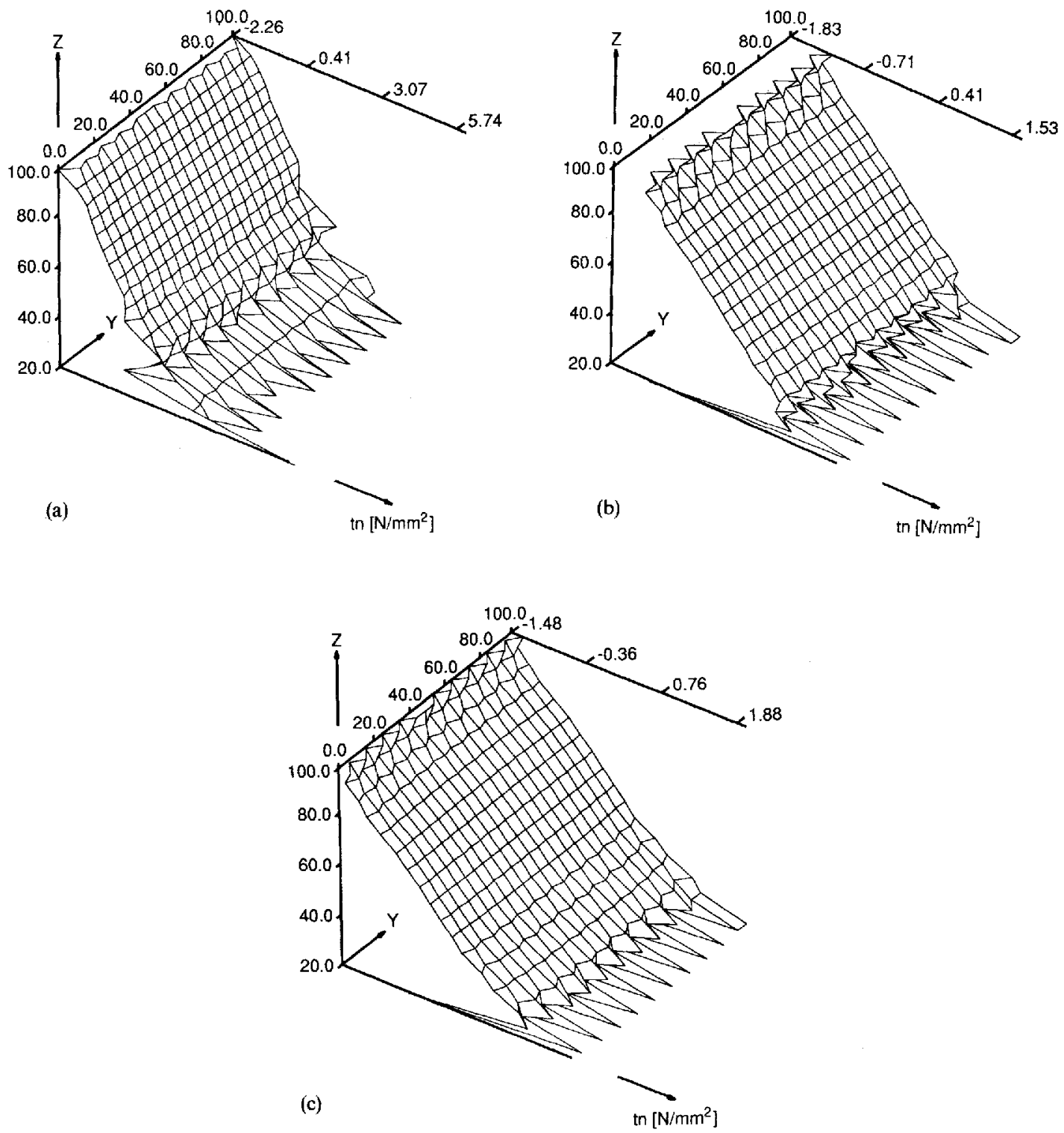

Figure 8. Traction profiles for quadratic plane interface elements with lumped integration: (a) $d_{n}=10^{+3}$; (b) $d_{n}=10^{+4}$; (c) $d_{n}=10^{+5}$

for this purpose is a pull-out test of a soil-reinforcement bar. The finite element mesh for the three-dimensional analysis is shown in Figure 9. In the mesh quadratic volume elements are used to model the soil mass and the reinforcement bar. In the analyses the horizontal and vertical displacements of the soil as well as the vertical displacements of the reinforcement bar were prevented. The interface is modelled with 16 -noded quadratic plane interface elements. The physically non-linear behaviour of the interface elements is governed by a Coulomb friction law

$$
\Phi=\sqrt{ }\left(t_{s}^{2}+t_{t}^{2}\right)+t_{n} \tan \phi-c
$$




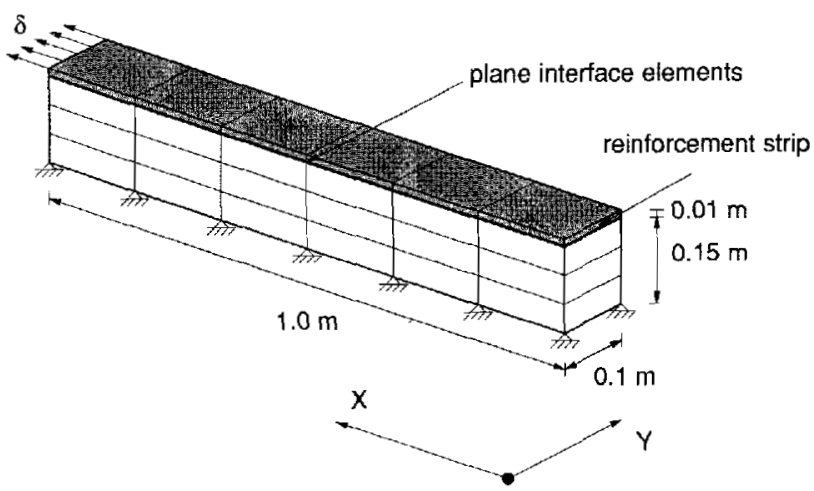

Figure 9. Finite element model of a three-dimensional soil-reinforcement system
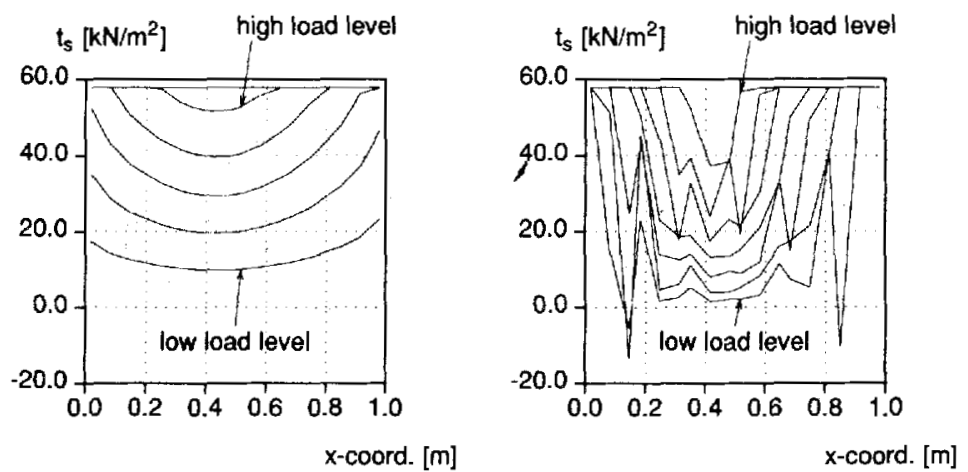

Figure 10. Shear traction profiles for quadratic plane interface elements with $3 \times 3$ Gauss integration. Left: $d_{s}=10^{+7}$. Right: $d_{s}=10^{+9}$

with $c$ the cohesion, $\phi$ the friction angle and $t_{i}$ the tractions. A more detailed description of the Coulomb friction model which includes a formulation of a consistent tangent operator has been given by Schellekens. ${ }^{21}$ In the analysis we have taken the cohesion equal to $0.577 \times 10^{+5} \mathrm{~N} / \mathrm{m}^{2}$ and the friction angle $\phi$ equal to zero. An associated slip law was used. For the stiffness values of the soil and reinforcement bar $E_{\text {soil }}=10^{+6} \mathrm{~N} / \mathrm{m}^{2}$ and $E_{\mathrm{re}}=10^{+9} \mathrm{~N} / \mathrm{m}^{2}$ have been substituted. The dummy stiffness for the interface elements was varicd between $10^{+7} \mathrm{~N} / \mathrm{m}^{3}$ and $10^{+9} \mathrm{~N} / \mathrm{m}^{3}$. The results from non-linear analyses with Newton-Cotes/Lobatto and Gauss integration schemes are given in Figures 10 and 11 and show that the Gauss scheme again results in an oscillating traction profile for the high dummy stiffness value of $10^{+9} \mathrm{~N} / \mathrm{m}^{3}$. Similar to the earlier observations the oscillations vanish with decreasing values for the dummy stiffness.

Sliding starts at $x=0 \mathrm{~m}$ and $x=1.0 \mathrm{~m}$ and propagates from the ends of the bar towards the centre. Figure 10 shows that oscillations appear just before the sliding front and that oscillations seem to disappear once the sliding front has passed. This phenomenon has also been observed by Gens et al. ${ }^{1}$ 

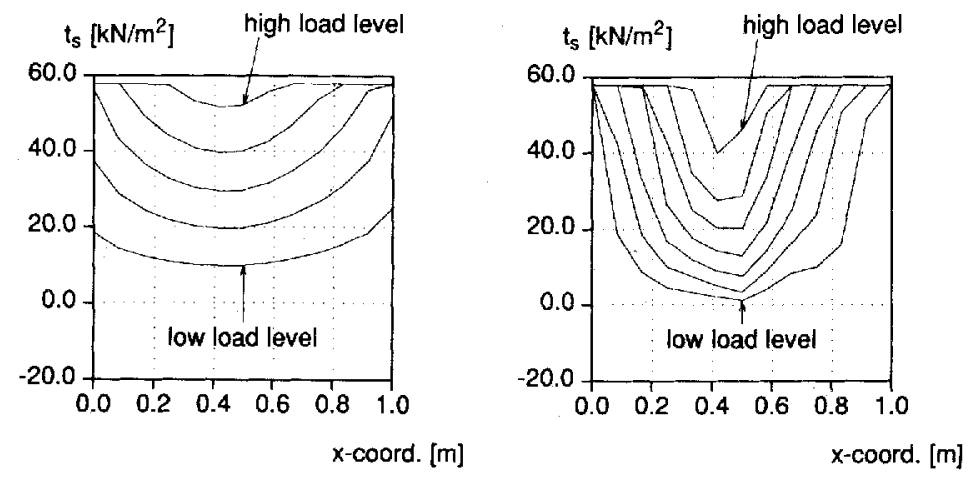

Figure 11. Shear traction profiles for quadratic plane interface elements with $3 \times 3$ Newton-Cotes/Lobatto integration. Left: $d_{s}=10^{+7}$. Right: $d_{s}=10^{+9}$

\section{EIGENVALUE ANALYSIS OF THE ELEMENT STIFFNESS MATRIX}

Eigenvalue analyses of the element stiffness matrix will now be presented to gain more insight in the underlying reasons for the oscillations in the traction profiles. For all four element types and for different integration schemes the non-zero eigenmodes and the corresponding eigenvalues of the linear elastic stiffness matrix are listed in Figures 12-15. The length, surface and dummy stiffness of the interface elements were taken equal to 1 . The eigenmodes of line interface elements and the linear plane interface elements do not show a coupling of nodal displacements between the individual node sets when integrated with a Newton-Cotes/Lobatto or a lumped integration scheme (Figures 12 and 13). However, such a coupling is observed when the same elements are integrated by a Gauss scheme. With regard to quadratic plane interface elements we observe that the eigenmodes of the element stiffness matrix possess coupling of displacements between node sets also for Newton-Cotes/Lobatto integration [Figure 15(b)], so that only lumped integration is free from this coupling of nodal displacements.

Furthermore, in Reference 21 eigenvalues and eigenmodes were calculated for the stiffness matrices of quadratic plane interface elements with a varying number of sliding points. These results show the effect of the progression of the non-linearity on the eigenvalues and eigenmodes. In particular, it was observed that a change in status of an integration point from elastic into sliding results in an increase of the number of uncoupled zero energy modes. Also, fully inelastic elements have no coupled non-zero energy modes left in the direction in which the elements show inelastic behaviour.

\section{INTERPRETATION AND EXPLANATION}

Before explaining the behaviour of the interface elements on the basis of the results of the eigenvalue analyses, clarity must exist about the conditions under which the element performance is not satisfactory. From the analyses of the concrete beam and the soil-reinforcement system we have seen that the introduction of a high dummy stiffness in combination with a Gauss integration scheme results in oscillations in the traction profiles. The cause of the problem, however, is not the high value of the dummy stiffness, but the resulting large traction gradient over an interface element. In the concrete beams such a high gradient occurs near the notch singularity and in the soil-reinforcement structure near the free ends of the reinforcement bar. It 


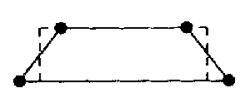

$\lambda=0.3333$

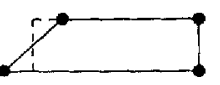

$\lambda=1.0$
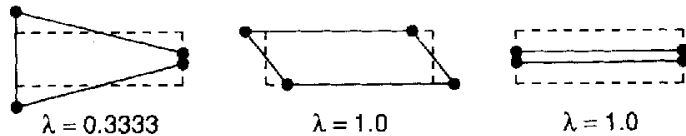

(a) 2-point Gauß integration scheme
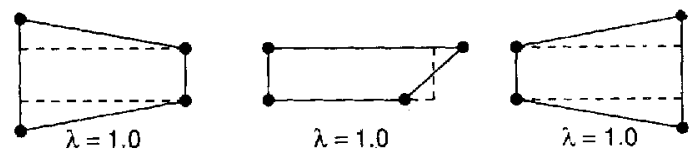

(b) 2-point Lobatto/Newton-Cotes or lumped integration

Figure 12. Eigenmodes and corresponding eigenvalues for a linear line interface element
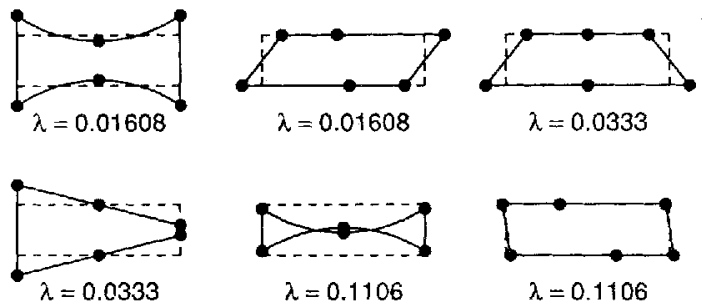

(a) 3-point Gauß integration
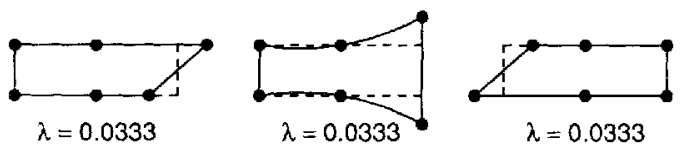

$\lambda=0.0333$
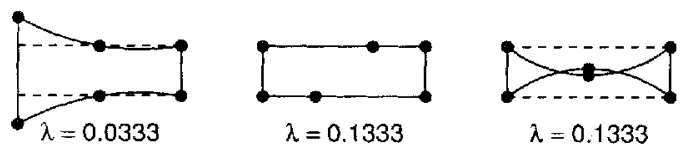

(b) 3-point Lobatto/Newton-Cotes or lumped integration

Figure 13. Eigenmodes and corresponding eigenvalues for a quadratic line interface element

appears that when a Gauss scheme is used the element is not able to describe properly highly inhomogeneous tractions within one single element. This is illustrated in Figure 16 which shows the effects of mesh refinement on the traction profile of the 3D soil-reinforcement problem. A $3 \times 3$ Gauss scheme is applied and the stiffness values $d_{s}$ and $d_{n}$ are equal to $10^{+9} \mathrm{~N} / \mathrm{m}^{3}$. The results are given for the cases with 20 and 40 elements over the length of the structure. We observe 

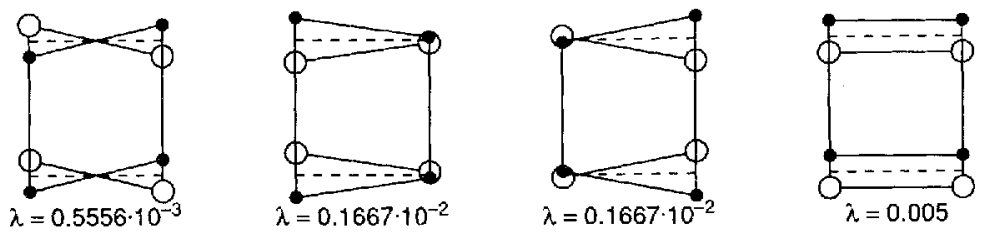

(a) $2 \times 2$ GauB integration
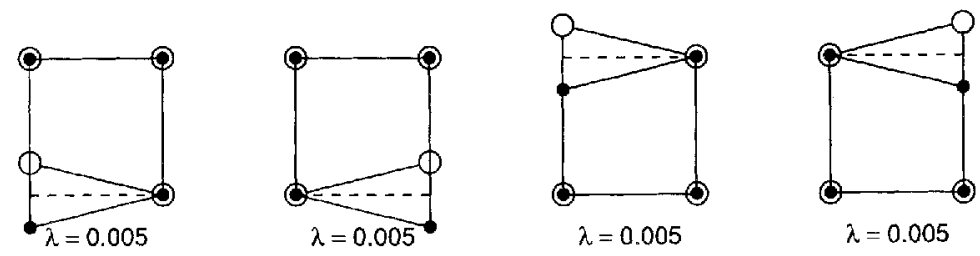

(b) $2 \times 2$ Lobatto, Newton-Cotes or lumped integration

Figure 14. Eigenmodes and corresponding eigenvalues for a linear plane interface element

that, with increasing mesh refinement, the element performance improves as a result of the decreasing traction gradients over a single element.

Accordingly, the combination of high traction gradients and the integration scheme is responsible for the improper element behaviour. In line and plane interface elements oscillations occur both in linear and non-linear analysis when Gauss integration is used. Newton-Cotes/Lobatto integration results in smooth traction profiles in all the analyses.

In contrast to Newton-Cotes/Lobatto integration, application of Gauss quadrature to line interface elements and linear plane interface elements results in the existence of non-zero energy modes with coupling between the degrees of freedom of the individual node sets. This causes oscillations in the traction profiles if a large traction gradient exists. Figure 15 (b) shows that for quadratic plane interface elements not only Gauss integration, but also Newton-Cotes/Lobatto integration results in non-zero energy modes with node set coupling. On the basis of this observation one would expect oscillatory traction profiles, which is not in agreement with our findings from the numerical experiments. In order to explain the good performance of Newton-Cotes/Lobatto integration schemes in the analyses, we shall further investigate the element stiffness matrix of the plane interface element. The linear elastic element stiffness matrix $\mathbf{K}$ assembled using Lobatto integration equals

$$
\mathbf{K}=\frac{1}{180} A\left[\begin{array}{rrrrrr}
\mathbf{K}_{n} & -\mathbf{K}_{n} & 0 & 0 & 0 & 0 \\
-\mathbf{K}_{n} & \mathbf{K}_{n} & 0 & 0 & 0 & 0 \\
0 & 0 & \mathbf{K}_{s} & -\mathbf{K}_{s} & 0 & 0 \\
0 & 0 & -\mathbf{K}_{s} & \mathbf{K}_{s} & 0 & 0 \\
0 & 0 & 0 & 0 & \mathbf{K}_{t} & -\mathbf{K}_{t} \\
0 & 0 & 0 & 0 & -\mathbf{K}_{t} & \mathbf{K}_{t}
\end{array}\right]
$$


in which each $\mathbf{K}_{i}$ for $i=n, s, t$ is an $8 \times 8$ submatrix of the form

$$
\mathbf{K}_{i}=\left[\begin{array}{rrrrrrrr}
10 d_{i} & -10 d_{i} & 5 d_{i} & -10 d_{i} & 5 d_{i} & -10 d_{i} & 5 d_{i} & -10 d_{i} \\
-10 d_{i} & 40 d_{i} & -10 d_{i} & 20 d_{i} & -10 d_{i} & 20 d_{i} & -10 d_{i} & 20 d_{i} \\
5 d_{i} & -10 d_{i} & 10 d_{i} & -10 d_{i} & 5 d_{i} & -10 d_{i} & 5 d_{i} & -10 d_{i} \\
-10 d_{i} & 20 d_{i} & -10 d_{i} & 40 d_{i} & -10 d_{i} & 20 d_{i} & -10 d_{i} & 20 d_{i} \\
5 d_{i} & -10 d_{i} & 5 d_{i} & -10 d_{i} & 10 d_{i} & -10 d_{i} & 5 d_{i} & -10 d_{i} \\
-10 d_{i} & 20 d_{i} & -10 d_{i} & 20 d_{i} & -10 d_{i} & 40 d_{i} & -10 d_{i} & 20 d_{i} \\
5 d_{i} & -10 d_{i} & 5 d_{i} & -10 d_{i} & 5 d_{i} & -10 d_{i} & 10 d_{i} & -10 d_{i} \\
-10 d_{i} & 20 d_{i} & -10 d_{i} & 20 d_{i} & -10 d_{i} & 20 d_{i} & -10 d_{i} & 40 d_{i}
\end{array}\right]
$$
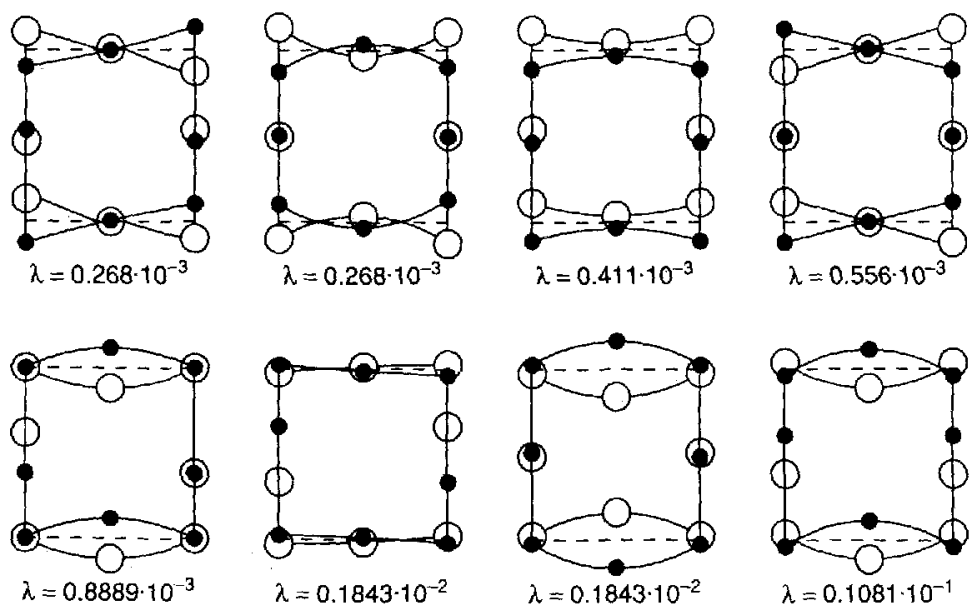

(a) $3 \times 3$ Gauß integration
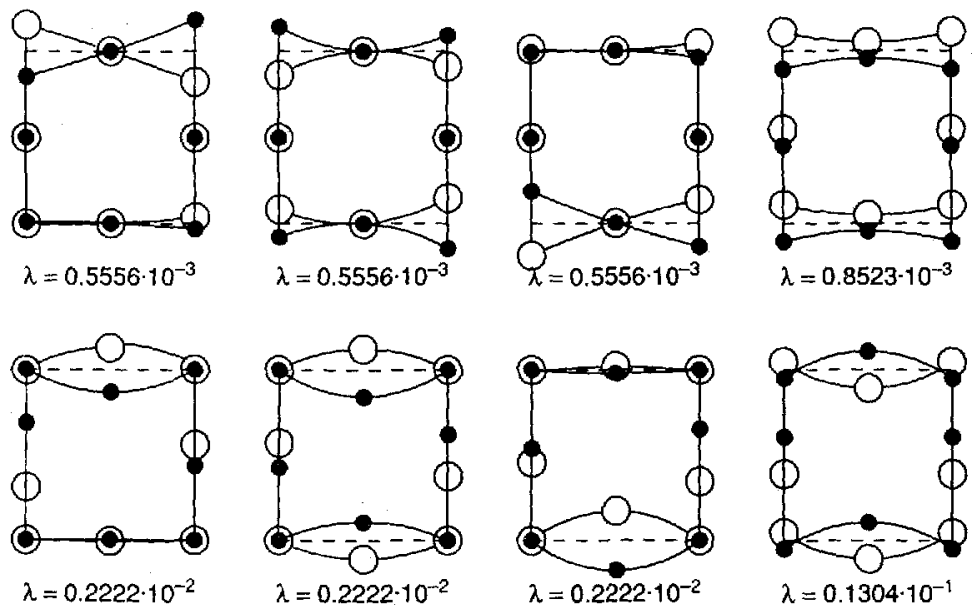

(b) $3 \times 3$ Newton-Cotes/Lobatto integration 

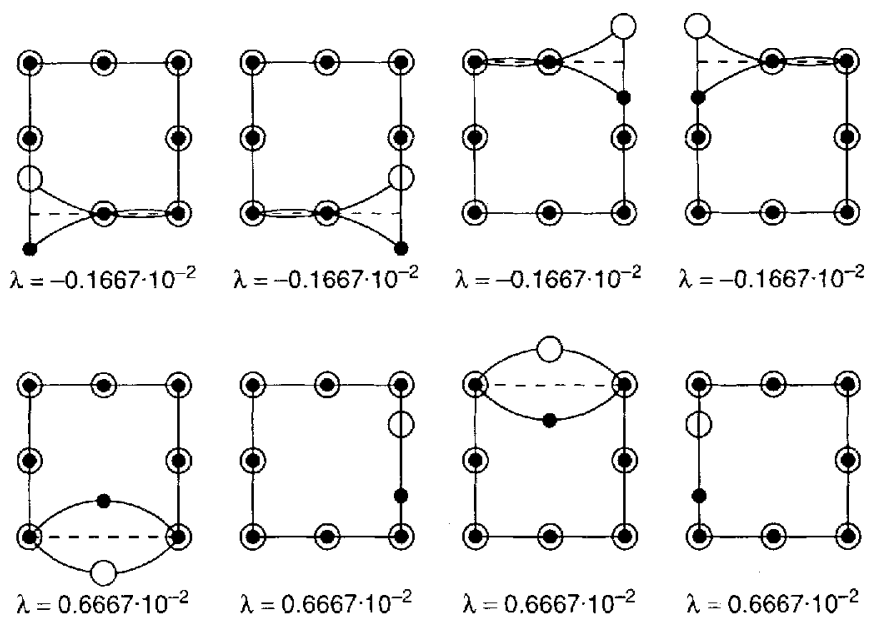

$\lambda=0.6667 \cdot 10^{-2}$

$\lambda=0.6667 \cdot 10^{-2}$

$\lambda=0.6667 \cdot 10^{-2}$

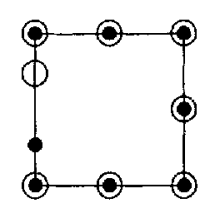

(c) Lumped integration

Figure 15. Eigenmodes and corresponding cigenvalues for a quadratic plane interface element: (a) $3 \times 3$ Gauss integration; (b) $3 \times 3$ Newton Cotes/Lobatto integration; (c) Lumped integration
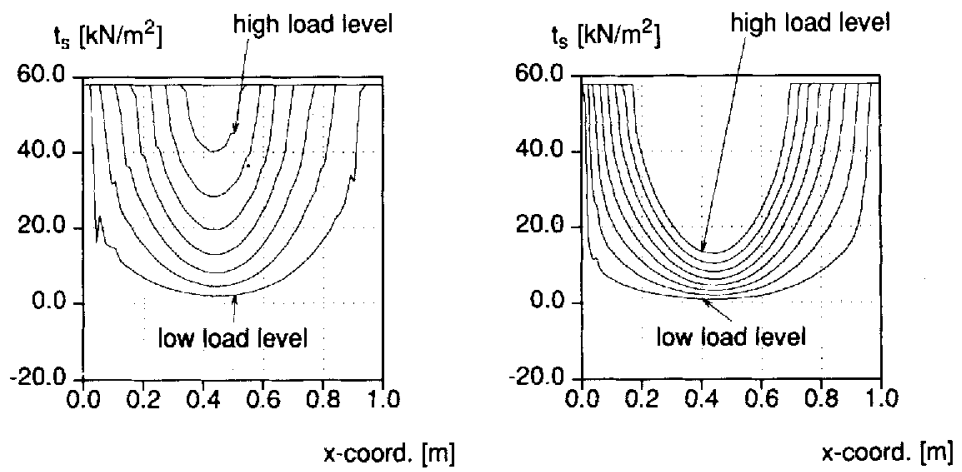

Figure 16. Shear traction profiles in soil-reinforcement structure for quadratic plane interface elements with $3 \times 3$ Gauss integration $\left(d_{s}=10^{+9}\right)$. Left: 20 elements. Right: 40 elements

If we decompose this submatrix in a matrix which contains the contributions of the integration points which coincide with the element nodes $\left(\mathbf{K}_{n}\right)$ and a matrix which contains the contribution to the stiffness matrix of the integration point located in the centre of the element $\left(\mathbf{K}_{m}\right)$ we obtain

$$
\mathbf{K}_{n}=\left[\begin{array}{rrrrrrrr}
5 d_{i} & 0 & 0 & 0 & 0 & 0 & 0 & 0 \\
0 & 20 d_{i} & 0 & 0 & 0 & 0 & 0 & 0 \\
0 & 0 & 5 d_{i} & 0 & 0 & 0 & 0 & 0 \\
0 & 0 & 0 & 20 d_{i} & 0 & 0 & 0 & 0 \\
0 & 0 & 0 & 0 & 5 d_{i} & 0 & 0 & 0 \\
0 & 0 & 0 & 0 & 0 & 20 d_{i} & 0 & 0 \\
0 & 0 & 0 & 0 & 0 & 0 & 5 d_{i} & 0 \\
0 & 0 & 0 & 0 & 0 & 0 & 0 & 20 d_{i}
\end{array}\right]
$$


and

$$
\mathbf{K}_{m}=\left[\begin{array}{rrrrrrrr}
5 d_{i} & -10 d_{i} & 5 d_{i} & -10 d_{i} & 5 d_{i} & -10 d_{i} & 5 d_{i} & -10 d_{i} \\
-10 d_{i} & 20 d_{i} & -10 d_{i} & 20 d_{i} & -10 d_{i} & 20 d_{i} & -10 d_{i} & 20 d_{i} \\
5 d_{i} & -10 d_{i} & 5 d_{i} & -10 d_{i} & 5 d_{i} & -10 d_{i} & 5 d_{i} & -10 d_{i} \\
-10 d_{i} & 20 d_{i} & -10 d_{i} & 20 d_{i} & -10 d_{i} & 20 d_{i} & -10 d_{i} & 20 d_{i} \\
5 d_{i} & -10 d_{i} & 5 d_{i} & -10 d_{i} & 5 d_{i} & -10 d_{i} & 5 d_{i} & -10 d_{i} \\
-10 d_{i} & 20 d_{i} & -10 d_{i} & 20 d_{i} & -10 d_{i} & 20 d_{i} & -10 d_{i} & 20 d_{i} \\
5 d_{i} & -10 d_{i} & 5 d_{i} & -10 d_{i} & 5 d_{i} & -10 d_{i} & 5 d_{i} & -10 d_{i} \\
-10 d_{i} & 20 d_{i} & -10 d_{i} & 20 d_{i} & -10 d_{i} & 20 d_{i} & -10 d_{i} & 20 d_{i}
\end{array}\right]
$$

From these matrices we see that coupling is indeed introduced by the centre integration point. We now introduce a characteristic part of the $\mathbf{B}$ matrix of the centre point which relates nodal displacements normal to the element to the normal relative displacement in the integration point.

$$
\mathbf{B}=\left[\begin{array}{cccccccc}
-0.25 & 0.5 & -0.25 & 0.5 & -0.25 & 0.5 & -0.25 & 0.5 \\
0 & 0 & 0 & 0 & 0 & 0 & 0 & 0 \\
0 & 0 & 0 & 0 & 0 & 0 & 0 & 0
\end{array}\right]
$$

The relation between the non-zcro components in the $\mathbf{B}$ matrix is such that when a displacement field over an element has a gradient in either the $\xi$-or $\eta$-direction, the nodes in the direction in which the displacement field varies, do not have a resulting contribution to the tractions and relative displacements in the central integration point. The tractions and relative displacements in the central point of the element are only dependent on the nodal values of the nodes which are located in the direction in which no gradient exists. So in these special cases the element acts as if no coupling between the node sets exists and no oscillations will occur. This can be assessed by a few examples. The upper side of an element is given in Figure 17. For the first problem, a linear varying displacement $\mathbf{u}_{n}=(1,1,1,0-5,0,0,0,0-5)$ is applied. The traction values in Figure 17 represent in a smooth traction profile. A simple calculation using equation (33) is sufficient to show that the traction value in the midpoint is the mean of the traction values of nodes 4 and 8 . The contributions of the other nodes cancel each other. If we apply a displacement field $\mathbf{u}_{n}=(1,0,0,0,0,0,0,0)$ that varies with $\xi$ and $\eta$, we observe that all the nodal displacements contribute to the relative displacement in the midpoint and that a non-smooth traction profile results (see Figure 17). As a conclusion we can state that, for quadratic plane interface clements, besides a few special cases (linear, quadratic and cubic variation in the displacement field from node 1 to 5). Newton-Cotes/Lobatto integration produces smooth traction profiles only when the displacement field over the element varies in only one direction. Since this was the case in the
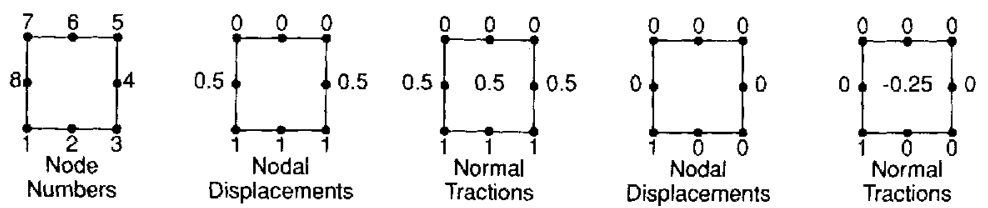

Figure 17. Preseribed nodal displacements and traction values for a Newton-Cotes/Lobatto integrated quadratic plane interface element 
presented examples no oscillatory traction profiles were encountered. In cases where Lobatto/Newton-Cotes integration gives oscillatory traction profiles one must either use extreme mesh refinement or apply linear elements. However, it is emphasized that, due to the fact that the tractions are calculated inaccurately only for the centre integration point, the spurious behaviour of Newton-Cotes/Lobatto integrated elements will be less severe than the spurious behaviour of Gaussian integrated elements. This is demonstrated next for two linear elastic analyses.

The first analysis is a modification of the three-dimensional beam analysis. In order to obtain a traction profile that varies in two directions an additional notch is provided (Figure 18) and the

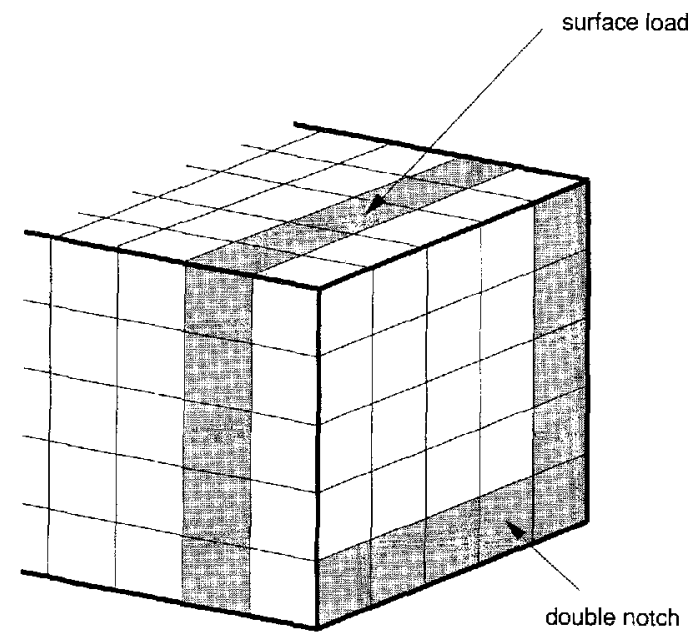

Figure 18. Double-notched three-dimensional beam loaded in two directions. The part of the mesh that is not shown is equal to the discretization in Figure 3

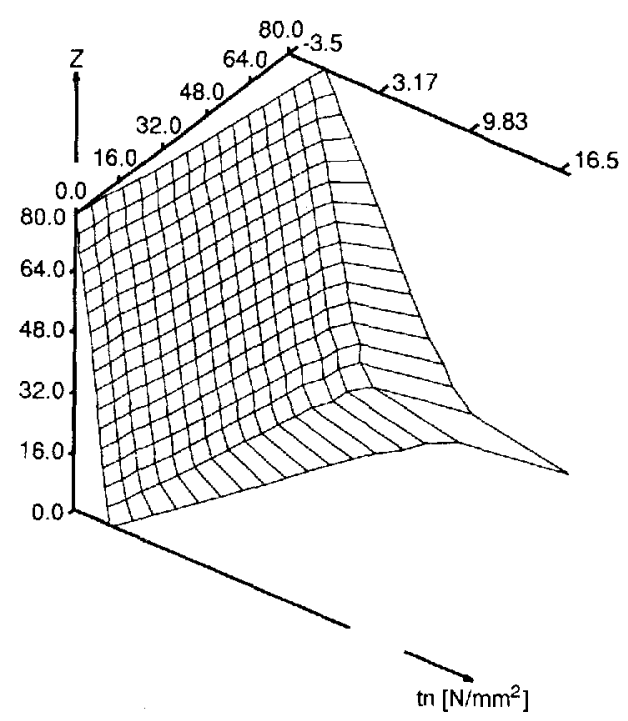

Figure 19. Traction profile for the double-notched three-dimensional beam. Quadratic plane interface elements with $3 \times 3$ Newton-Cotes/Lobatto integration 
beam is loaded in the horizontal and vertical direction. The dimensions and material properties in this analysis are as specified in Section 3.2. The dummy stiffness of the quadratic plane interface elements is set equal to $d_{n}=10^{+9} \mathrm{~N} / \mathrm{m}^{3}$. From Figure 19 which shows the traction surface for a $3 \times 3$ Newton Cotes/Lobatto integration scheme we conclude that the interface performance is still satisfactory, even under more complex loading conditions.

A linear elastic analysis of the soil-reinforcement system presented in Figure 20 was used to demonstrate the element performance under non-uniform shear loading. The reinforcement plate was loaded in the $x$ - and $y$-direction by uniform surface loads equal to $3.0 \times 10^{+6} \mathrm{~N} / \mathrm{m}^{2}$ and $1 \cdot 0 \times 10^{+6} \mathrm{~N} / \mathrm{m}^{2}$, respectively. The dummy stiffness values of the interface elements were assumed to be $d_{s}=d_{t}=10^{+9} \mathrm{~N} / \mathrm{m}^{3}$. The other material properties were equal to the values specified in Section 3.3. Figures 21 and 22 show the results for $3 \times 3$ Gaussian integration and $3 \times 3$ Newton-Cotes/Lobatto integration. Again we observe a good performance of the Newton-Cotes/Lobatto integrated interface elements, especially when compared to the results obtained from Gaussian integration.

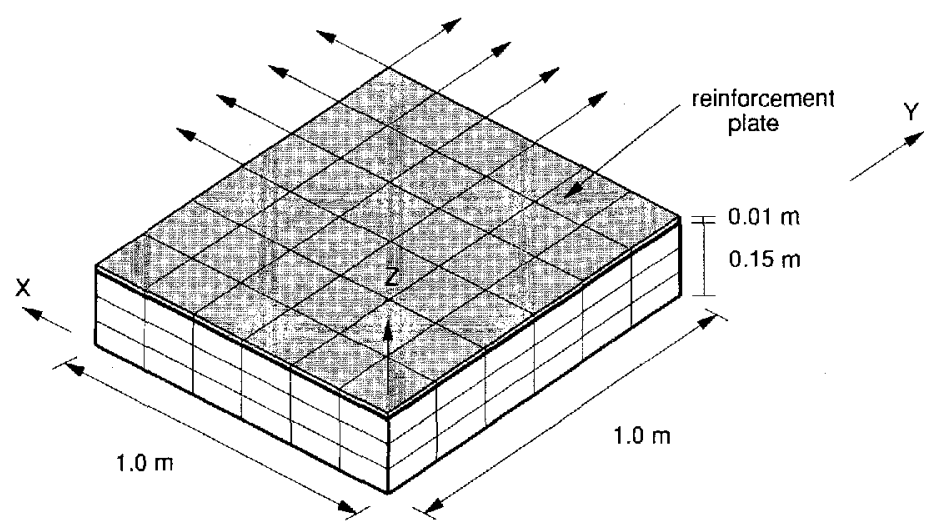

Figure 20. Soil-reinforcement system loaded in shear in two directions
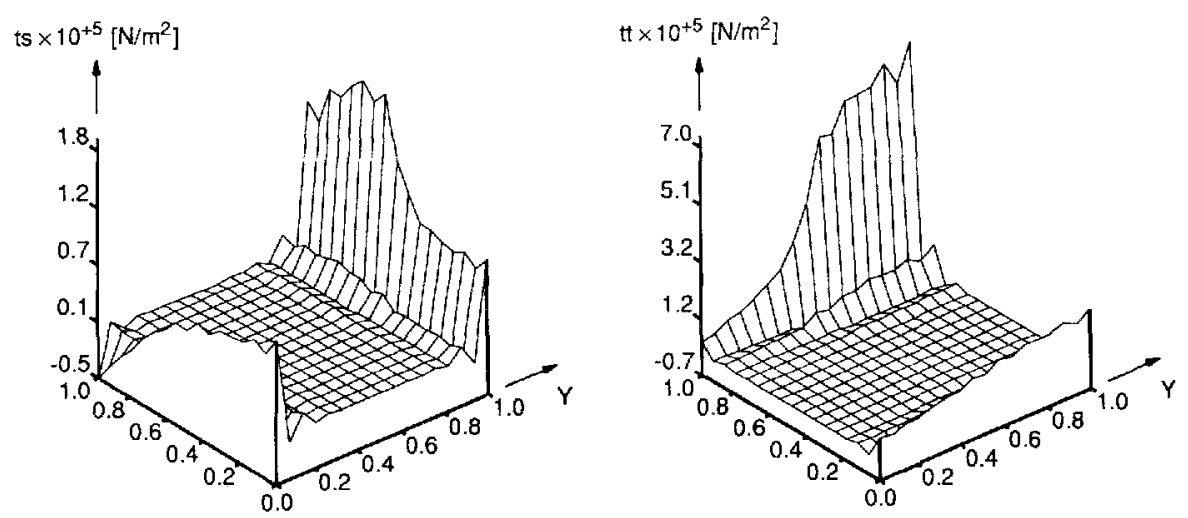

Figure 21. Shear tractions for soil-reinforcement system when applying $3 \times 3$ Gauss integration $\left(d_{s}=10^{+9}\right)$. Left: in $y$-direction. Right:in $x$-direction 

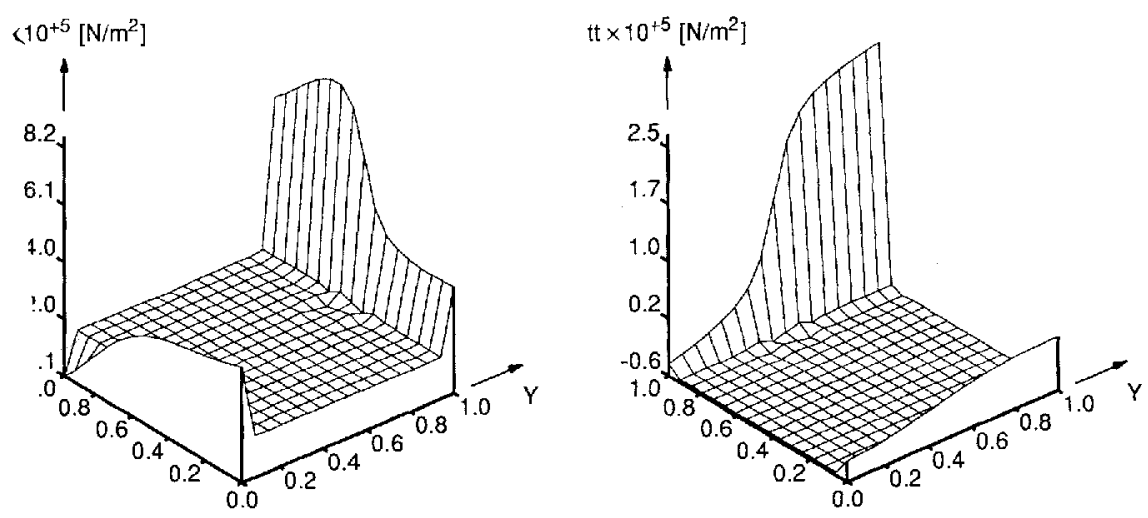

Figure 22. Shear tractions for soil-reinforcement system when applying $3 \times 3$ Lobatto integration $\left(d_{s}=10^{+9}\right)$. Left: in $y$-direction. Right: in $x$-direction

\section{CONCLUDING REMARKS}

Preliminary calculations indicate that the application of linear distorted plane interface elements produces inaccurate results when a Newton-Cotes/Lobatto integration scheme is used to assemble the element stiffness matrix. This is caused by an improper calculation of the surface contributions (det $\mathbf{J}$ ) for the integration points. Elements that are integrated by a Gaussian integration scheme or a nodal lumping scheme do not suffer from this deficiency.

\section{ACKNOWLEDGEMENTS}

The calculations in this report are performed with the DIANA finite element package of TNO Building and Construction Research on a Sun SPARC station 1 of the Delft University of Technology.

\section{REFERENCES}

1. A. Gens, I. Carol and E. E. Alonso, 'An interface element formulation for the analysis of soil-reinforcement interaction', Comput. Geotech., 7, 133-151 (1988).

2. J. M. Hohberg and H. Bachmann, 'A macro joint element for nonlinear arch dam analysis', in G. Swoboda (ed.), Numerical Methods in Geomechanics, Balkema, Rotterdam, 1989, pp. 829-834.

3. B. Weber, J. M. Hohberg and H. Bachmann, 'Earthquake analysis of arch dams including joint non-linearity and fluid/structure interaction', Dam Eng., 1, 267-278 (1990).

4. E. E. Alonso and I. Carol, 'Foundation analysis of an arch dam. Comparison of two modelling techniques: No tension and jointed rock material', Rock Mech. Rock Eng., 18, 149-182 (1985).

5. R. E. Goodman, R. L. Taylor and T. L. Brekke, 'A model for the mechanics of jointed rock', ASCE J. Soil Mech. Found. Div., 94, 637-659 (1968).

6. A. Gens, I. Carol and E. E. Alonso, 'Elasto-plastic model for joints and interfaces', in D. R. J. Owen, E. Hinton and E. Onate (eds.), Proc. 2nd Int. Conf. Computational Plasticity, Vol. 2, Pineridge Press, Swansea, 1989, pp. 1251-1264.

7. J. G. Rots, 'Computational modeling of concrete fracture', Dissertation, Delft University of Technology, Delft, 1988,

8. J. Janssen, 'Mode-I fracture of plain concrete under monotonic and cyclic loading', Report 25.2-90-2-05, Delft University of Technology, Delft, 1990.

9. P. H. Feenstra, R. de Borst and J. G. Rots, 'A numerical study on crack dilatancy: I and II', ASCE J. Eng. Mech., 117, 737-769 (1991).

10. D. Ngo and A. C. Scordelis, 'Finite element analysis of reinforced concrete beams', J. Am. Concr. Inst., 64, 152-163 (1967).

11. M. Keuser, G. Mehlhorn and V. Cornelius, 'Bond between prestressed steel and concrete: Computer analysis using ADINA', Comp. Struct., 17, 669676 (1983). 
12. G. Mehlhorn, J. Kollegger, M. Keuser and W. Kolmar, "Nonlinear contact problems-A finite element approach implemented in ADINA', Comp. Struct, 21, 69-80 (1985).

13. G. Mehlhorn and M. Keuser, 'Isoparametric contact elements for analysis of reinforced concrete structures', in C. Meyer and H. Okamura (eds.), Finite Element Analysis of Reinforced Concrete Structures, ASCE, New York, 1985 , pp. 329-347.

14. H. Schäfer, 'A contribution to the solution of contact problems with the aid of bond elements', Comp. Methods Appl. Mech. Eng., 6, 335-354 (1975).

15. J. C. J. Schellekens and R. de Borst, Numerical simulation of free edge delamination in graphite-epoxy specimen under uniaxial tension', in I. H. Marshall (ed.), Composite Structures 6, Elsevier, London and New York, 1991, pp. $647-657$.

16. J. C. J. Schellekens and R. de Borst, 'Nonlinear analysis of propagation of delamination near free edges', in S. W. Tsai and G. S. Springer (eds.), Composites: Design, Manffacture and Application, SAMPE, Covina, California, 1991, Paper E-28.

17. T. Rodic and D. R. J. Owen, 'A plasticity theory of friction and joint elements', in D. R. J. Owen, E. Hinton and E. Onate (eds.), Proc. 2nd Int. Conf. Computational Plasticity, Vol. 2, Pineridge Press, Swansea, 1989, pp. $1043-1062$.

18. G. Beer, 'An isoparametric joint/interface element for finite element analysis', Int, $j$. numer. methods eng., 21, 585-600 (1985).

19. O. C. Zienkiewicz, The Finite Element Method, McGraw-Hill, London, 1977.

20. J. G. Rots and J. C. J. Schellekens, 'Interface elements in concrete mechanics', in N. Bićanić and H. Mang (eds.), Computer Aided Analysis and Design of Concrete Structures, Pincridge Press, Swansea, 1990, pp. 909-918.

21. J. C. J. Schellekens, 'Interface elements in finite element analysis', Report 25.2-90-2-17, Delft University of Technology, Delft, 1990.

22. J. M. Hohberg, 'A note on the spurious kinematic oscillations in FEM joint elements', Earthquake eng. struct. dyn., 19, $773-779(1990)$

23. X. Qiu, M. E. Plesha and D. W. Meyer, 'Stiffness matrix integration rules for contact-friction finite elements', Comp. Methods Appl. Mech. Eny., to appear. 